\title{
Comparing Sequential and Temporal Patterns from Human Mobility Data for Next-Place Prediction
}

\author{
Yunlong Wang ${ }^{1}$, Corinna Breitinger ${ }^{1}$, Björn Sommer ${ }^{2}$, Falk Schreiber ${ }^{2}$, Harald Reiterer ${ }^{1}$ \\ ${ }^{1}$ Human Computer Interaction Group, University of Konstanz, Konstanz, Germany \\ ${ }^{2}$ Computational Life Sciences Group, University of Konstanz, Konstanz, German \\ \{yunlong.wang, corinna.breitinger, bjoern.sommer, falk.schreiber, harald.reiterer\}@uni-konstanz.de
}

\begin{abstract}
In the domain of human behavior prediction, next-place prediction is an active research field. While prior work has applied sequential and temporal patterns for next-place prediction, no work has yet studied the prediction performance of combining sequential with temporal patterns compared to using them separately. In this paper, we address next-place prediction using the sequential and temporal patterns embedded in human mobility data that has been collected using the GPS sensor of smartphones. We test five next-place prediction methods, including single pattern-based methods and hybrid methods that combine temporal and sequential patterns. Instead of only examining average accuracy as in related work, we additionally evaluate the selected methods using incremental-prediction accuracy on two publicly available datasets (the MDC dataset and the StudentLife dataset). Our results suggest that (1) integrating multiple patterns is not necessarily more effective than using single patterns in average prediction accuracy, (2) most of the tested methods can outperform others for a certain time period (either for the prediction of all places or each place individually), and (3) average prediction accuracies of the top-three candidates using sequential patterns are relatively high (up to 0.77 and 0.91 in the median for both datasets). For real-time applications, we recommend applying multiple methods in parallel and choosing the prediction of the best method according to incrementalprediction accuracy. Lastly, we present an expert tool for visualizing the prediction results.
\end{abstract}

\section{CCS CONCEPTS}

- Human-centered computing $\rightarrow$ Ubiquitous and mobile computing

\section{KEYWORDS}

Next-place prediction, personalized and adaptive prediction, human mobility, first-order Markov chain, Active LeZi

\section{INTRODUCTION}

In many mobile health [28] applications, recommendations should be delivered to users before the target behavior occurs. Therefore, human behavior prediction plays a very important role. For example, Rahman et al. predicted the "about-to-eat" event to design food intake interventions. In the research field of just-intime adaptive interventions (JITAIs), the prediction of the opportune moments [19] or meaningful moments [27] determines both the effectiveness of the interventions and the user experience. Among human behavior prediction studies, locationbased applications are drawing much attention, since human behaviors are usually coupled with specific places and the corresponding information is relatively easy to collect using smartphones [17, 20].

In the domain of human behavior prediction, next-place prediction is currently an active research field $[6,10,15,31]$. To our knowledge, there is no study applying next-place prediction to mobile health applications. We see high potential of using next-place prediction to provide personalized and adaptive mobile health interventions and recommendations. Next-place prediction can be regarded as a contextual prediction problem where the next place is assumed to depend on a user's context of current and previous states [5]. The context refers to any information related to the prediction (e.g., the user's routine, calendar events, weather forecast). However, context information is not always available in practice due to privacy concerns or other limitations (e.g., manual logging). In this work, we focus on sequential and temporal patterns that can be derived directly from the past trajectories of human mobility.

Sequential patterns stand for repeated sub-sequences appearing in a specific order within a given sequence, e.g., Alice often ( 8 out of 10 times) visits place B after place A according to her previous month's mobility data. Temporal patterns refer to the time distribution for visited places. For instance, Bob arrives 


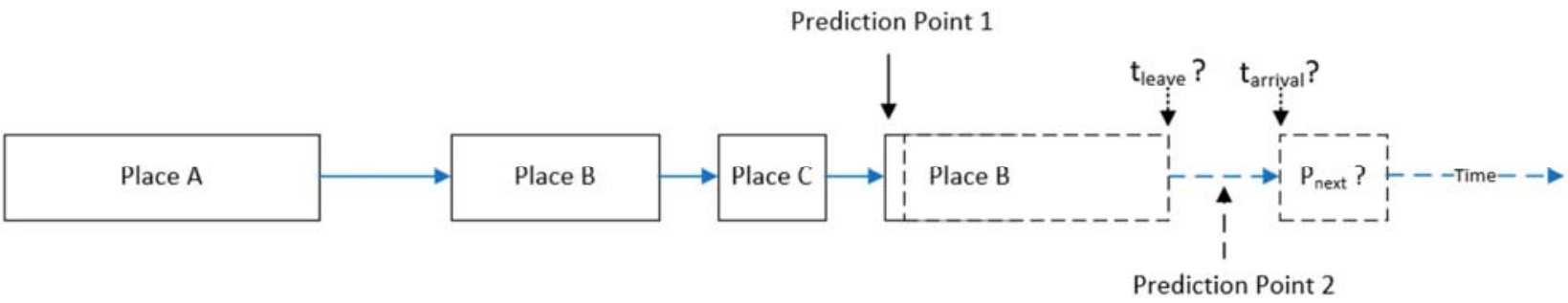

Figure 1: The human mobility model, containing concrete person's locations (continuous boxes) and predicted ones (dashed boxes), as adapted from [10].

at his office between 8:30 am and 9:00 am on Mondays (3 out of 4 times) given his previous month's mobility data.

In a recent work, Ikanovic and Mollgaard [9] examined the limits of predictability in human mobility using GPS traces collected by smartphones from 604 individuals in Denmark. The upper limit of predictability for next-place prediction using the method by Song et al. [23] was $71.1 \%$, while the average prediction accuracy using a first-order Markov chain was 39.8\%. Although Song et al. [24] argue that the upper limit calculated by their method is in principle attainable by an appropriate algorithm, such an algorithm has not been found yet. However, we can still use the predictability limit to compare how far an algorithm's performance is away from the theoretical limit. In addition to using a first-order Markov chain as in [9], we compare the performance of a more sophisticated Markov chain-based model, Active LeZi, to the predictability limit using two publicly available datasets, the Mobile Data Challenge (MDC) [11, 13] dataset and the StudentLife dataset [25].

Markov chain-based models only consider sequential patterns. In the methods we evaluate, we also consider a temporal patternbased method inspired by the work of Khoroshevsky and Lerner [10]. Although prior work has already used sequential and temporal patterns for next-place prediction, the performance of combining both sequential and temporal patterns compared to using single patterns has not yet been studied. In our study, we include not only three single pattern-based methods but also two sequential and temporal hybrid methods using joint conditional probability. Thus, our study allows comparing the prediction performance between these methods for the first time.

Another missing component in related work is that only the prediction performance on average among all users' data has been analyzed. To provide an in-depth analysis on the prediction results at the level of the individual and even at the level of the Places of Interest (POI) over time, we use incremental-prediction accuracy and develop an interactive visualization tool to present the evaluation results.

In this work, we examine the following research questions:

(Q1) What is the prediction performance of using the selected sequential and temporal patterns separately?

(Q2) What is the prediction performance of using sequential patterns and temporal patterns in combination based on joint conditional probability?

(Q3) Can any method outperform other methods for all of the users' data on average?

(Q4) Can any method outperform other methods for all places or for one specific place at the level of the individual, as well as at the POI level over time?

The remainder of this paper is structured as follows. In Sect. 2, we clarify two models of human mobility from the literature and describe the prediction task based on our selected model. Subsequently, we present related work (Sect. 3), before describing our methodology to address the stated research questions (Sect. 4). In Sect. 5, we present the evaluation and report on our results. Then, we introduce our visualization tool for analyzing the results in more detail, followed by a discussion of the contributions and drawbacks of our evaluation. Finally, we conclude the paper and point out future work.

\section{Problem Statement}

The spatio-temporal sequential data of a person's mobility trajectory can be illustrated as shown in Figure 1. Blocks represent places where a person spends a period of time, blocks with dashed shapes illustrate predicted ones, while blue arrows show transitions between these places. Places are extracted from GPS traces using clustering algorithms as in $[10,26,30]$ and mapped into symbol sequences as shown in Table 1.

Given an individual's historical mobility data and current state (i.e. place and time), the prediction problem thus encompasses the following sub-questions:

(1) What is the predicted leaving time from the current place (tleave in Figure 1)?

(2) What will be the next visit place $\left(P_{\text {next }}\right.$ in Figure 1)?

(3) What will be the arrival time at $P_{\text {next }}$ ( $t_{\text {arrival }}$ in Figure 1)?

As we can see in Figure 1, if a person is currently in transition

Table 1: A symbolic-temporal sequence representing human mobility [11].

\begin{tabular}{l|c|c|c|c|c}
\hline Place & A & B & C & B & C \\
\hline Arrival Time & $2017-1-89: 00$ & $2017-1-810: 20$ & $2017-1-811: 10$ & $2017-1-811: 40$ & $2017-1-813: 00$ \\
\hline Departure Time & $2017-1-810: 00$ & $2017-1-811: 00$ & $2017-1-811: 30$ & $2017-1-812: 40$ & $2017-1-814: 00$ \\
\hline
\end{tabular}


Table 2: Time bin-based symbolic-temporal sequence (based on the sequence in Table 1).

\begin{tabular}{c|c|c|c|c|c|c|c|c|c|c|c|c}
\hline Place & A & A & A & A & B & B & B & C & B & B & B & B \\
\hline Time & $\begin{array}{c}2017-1-8 \\
9: 00\end{array}$ & $9: 20$ & $9: 40$ & $10: 00$ & $10: 20$ & $10: 40$ & $11: 00$ & $11: 20$ & $11: 40$ & $12: 00$ & $12: 20$ & $12: 40$ \\
\hline
\end{tabular}

between two places (Prediction Point 2 in Figure 1), only question 2 and 3 should be answered. In this case, next-place prediction is easier because more information is provided (e.g., moving direction). In this paper, we focus on question 2 at Prediction Point 1 as shown in Figure 1.

The data model we adopt is in line with [9], where the next place is always different from the current one. In comparison, another data model (shown in Table 2) represents a time binbased sequence. We can see that this model contains continuous duplicates of places $[5,16,23]$. With such a data model, nextplace prediction is equivalent to next-bin prediction, which means that each place is computed for the next time bin featuring an individual duration [9]. The prediction accuracy in this model is greatly affected by the size of the time bin. When the time bin size is very small (e.g., one minute), the prediction accuracy will be very high by just using the current place as the prediction of the place in the next time bin. However, this high prediction accuracy only shows that the individual has stayed at the same place. It can be difficult to choose the appropriate size of the time bin in practice. Once the size of the time bin has been chosen, the time scale is fixed, which cannot be flexibly adjusted in practice.

\section{RELATED WORK}

\subsection{Sequential Patterns}

The simplest model of sequential patterns is a first-order Markov chain (MC1), which assumes that the prediction of the next place is only related to the current one. This method is often used as a naive baseline method when evaluating other more sophisticated methods $[9,10,18]$. We also include this method in our evaluation.

Unlike fixed-order Markov models, such as MC1, Active LeZi is an incremental parsing algorithm that can extract sequential patterns of different lengths [8]. Stemming from the family of LZ-based data compression algorithms [1, 21, 32], Active LeZi constructs a tree to contain the sequential patterns. Active LeZi solves the problems of ignoring cross-boundary patterns and embedded patterns known from previous LZ-based algorithms [21]. In addition, Active LeZi adopts a partial prediction match (PPM) strategy [4] for probability assignment to solve the zerofrequency problem. Another property of Active LeZi is that longer sequential patterns are weighted higher than shorter patterns. The reason is that longer sequential patterns appear later in the sequence, i.e. the more recent patterns have more impact on the current prediction when mapped to next-place prediction. While Active LeZi has shown promising prediction accuracy when applied in the smart home setting [8] and for human mobility at the cellular scale [3], no prior work has examined
Active LeZi when applied to next-place prediction on the more granular GPS data collected by smartphones in a real-world outdoor setting.

\subsection{Temporal Patterns}

Compared to sequential patterns, temporal patterns (e.g., visiting time and day of week) are more intuitive because they can be understood as schedules. For example, Cindy, is an office worker. During weekdays, she goes to work at 9:00 am and leaves her office at 5:00 pm. Khoroshevsky and Lerner [10] included five temporal pattern-based methods in their evaluation, which use the place: (1) with the highest conditional probability given the day of the week; (2) with a non-zero conditional probability given the day of the week, while being spatially nearest to the current place; (3) with a non-zero conditional probability given the hour of the day and being temporally closest to the current time; (4) with the highest conditional probability given the day of the week and the hour of the day; (5) with a non-zero conditional probability given both the day of the week and the hour of the day, while being spatially the closest to the current place. Surprisingly, the average prediction accuracies $(44-46.5 \%)$ were much lower than when using $\mathrm{MC1}(70.25 \%)$ [10]. Do et al. used several temporal patterns including time-of-day (similar to (4)), day-of-week (same as (1)), is-weekend, and temporally close places in their study [5]. However, none of the temporal patterns were tested separately. We include a temporal pattern (see Sect. 4 for details) in our evaluation based on (1) and (4).

\subsection{Combining Sequential and Temporal Patterns}

We can see that the sequential and temporal patterns mentioned above are all based on our understanding of daily human routines. For example, if asked to predict where a friend (Bob) will go next, we may say 'to the gym' if we know that he often visits the gym on Tuesday evenings or after staying at home for the whole afternoon. However, human behaviors are diverse, and we cannot rely on any single set of rules to explain and predict all behaviors. Bayesian inference [5], random forest [10], and embedding theorem of non-linear time series analysis [22] were used in previous works to combine patterns to improve prediction accuracy. The method based on random forest in [10] does not beat the MC1-based method. In [5], several temporal patterns are combined using Bayesian inference. The results show that the prediction accuracy increases when combining more patterns. However, Do et al. [5] did not include sequential patterns, and additionally, they adopted a next time bin model (see Sect. 2) instead of the next-place model used in this paper. 


\section{METHODOLOGY}

To answer the four research questions, we select three single pattern-based methods (one temporal pattern and two sequential patterns) and two hybrid methods combining the single patterns. Figure 2 shows the five methods to be compared in our evaluation: (1) The visiting time distribution of each place for the given time of day and day of the week (Temporal); (2) MC1; (3) Active LeZi; (4) Joint conditional probability with both (1) and (2) (Temporal+MC1); (5) Joint conditional probability with both (1) and (3) (Temporal+Active LeZi).

Method (1) represents the only temporal pattern-based method in our evaluation. Given the current time in minutes of a day $\left(t_{a}\right)$ and day of the week, we calculate the visiting frequencies of each candidate place on this day of the week after $t_{a}$ in the current day to get the conditional probabilities $\left(P_{i}=P\left(l_{i} \mid \mathrm{c}=t_{a}\right)\right)$ for POI $l_{i}$. We select the place with the highest visiting frequency as the prediction. If any $P_{i}$ is zero, we assume that the user stays in the current place for the rest of the given day (e.g., home) and we then use the next day of the week to calculate the conditional probabilities. Method (2) and (3) use the first-order Markov chain and Active LeZi, respectively, which have been described in Sect 3.1.

$$
\arg \max _{i} P\left(l_{i} \mid c\right)
$$

As shown in Formula (1), method (1), (2) and (3) choose the POI $l_{i}$ that maximizes the conditional probability $P\left(l_{i} \mid \mathrm{c}\right)$ as the prediction. The $c$ refers to the temporal or sequencial pattern.

\begin{tabular}{c|c|c}
\hline $\begin{array}{c}\text { First-order Markov } \\
\text { Chain } \\
\text { (MC1) }\end{array}$ & $\begin{array}{c}\text { Visiting Time Distribution on } \\
\text { Each Day of the Week } \\
\text { (Temporal) }\end{array}$ & $\begin{array}{c}\text { Active LeZi } \\
\text { (ALZ) }\end{array}$ \\
\hline (2) & (1) & (3) \\
& (4) &
\end{tabular}

Figure 2: The five selected methods in our evaluation.

Method (4) and (5) combine the conditional probability based on the temporal pattern with those based on the two sequential patterns. Under the naïve assumption that the temporal pattern and sequential patterns are independent of each other, we calculate the joint conditional probability using Bayes' theorem as shown in Equation (2).

$$
\begin{gathered}
P(l \mid \boldsymbol{c})=\frac{P(\boldsymbol{c} \mid l) P(l)}{P(\boldsymbol{c})}=\frac{\left(\prod_{i}^{N} P\left(c_{i} \mid l\right)\right) P(l)}{\prod_{i}^{N} P\left(c_{i}\right)}=\frac{\prod_{i}^{N}\left(\frac{P\left(c_{i} \mid l\right) P(l)}{P\left(c_{i}\right)}\right)}{(P(l))^{N-1}} \\
=\frac{\prod_{i}^{N}\left(P\left(l \mid c_{i}\right)\right)}{(P(l))^{N-1}}
\end{gathered}
$$

The POI with the maximum joint conditional probability $\left(P\left(l_{j} \mid \mathbf{c}\right)\right)$ is regarded as the prediction as shown in Formula (3), where $l_{j}$ refers to the $\mathrm{j}$-th prediction candidate.

$$
\arg \max _{j} P\left(l_{j} \mid \mathbf{c}\right)
$$

\section{EVALUATION}

This section presents the evaluation results of the selected methods. We begin by describing the datasets, and then introduce the incremental-prediction accuracy and discuss the predictability limit. All the methods were implemented and evaluated in Matlab 2017a.

\subsection{Datasets}

The two datasets used in our evaluation are the Mobile Data Challenge $(M D C)$ dataset $[11,13]$ and the StudentLife dataset [25]. Both datasets contain daily life mobility data collected by smartphones over a 24-hour period, seven days per week. The data collection duration in the MDC dataset is 18 months and thus much longer than in the StudentLife dataset (10 weeks). To make the MDC dataset comparable to the StudentLife dataset, we exclusively selected the participants' data from the MDC dataset covering more than 60 days, which resulted in a dataset containing data from 144 participants. Moreover, we only kept 60 days worth of data for each participant for this dataset.

We obtained the sequences of POIs based on these two datasets using the POI clustering method proposed in [26]. Each user's mobility is modeled as one sequence of POIs, as shown in Table 1. We use the term sequences for short to indicate 'sequences of POIs' in the remainder of this section.

Since prediction results strongly depend on the quality of the extracted sequences [10], we first filter the sequences according to the following criteria: (1) sequences containing less than three or more than 90 unique places are ignored; (2) sequences with a length shorter than 50 or over 3,000 are excluded. A very small number of unique places or a short sequence length indicate that the sequences might miss significant mobility data. On the other hand, a very large number of unique places or long sequence length can indicate that the sequences contain too much noise. We apply our criteria to the resulting 49 sequences from the StudentLife dataset and the 144 ones from the MDC dataset, respectively. Twelve sequences had less than three unique places, while thirteen sequences were longer than 3,000. Finally, 47 sequences from StudentLife dataset and 71 sequences from MDC dataset were kept for evaluation.
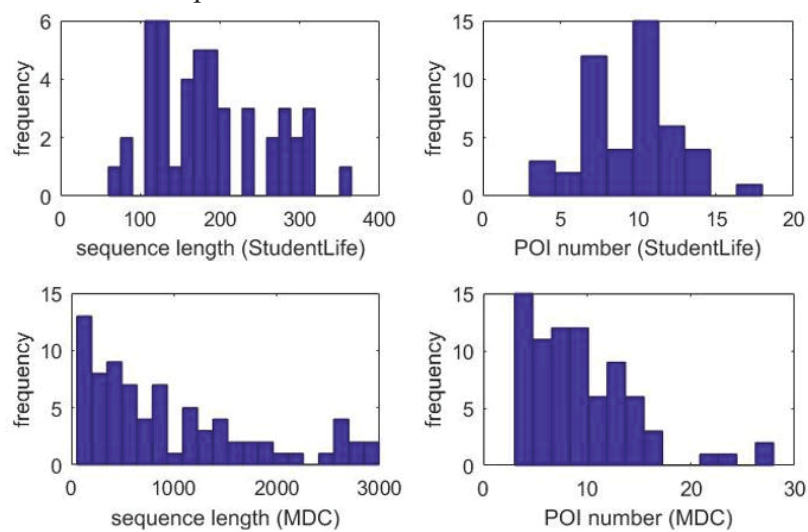

Figure 3: Distribution of sequence length and POI number of the prepared data from the StudentLife dataset and MDC dataset. 
Figure 3 shows the distribution of sequence lengths and the number of unique places for the selected sequences from both datasets. The majority of sequences from the StudentLife dataset have 100-300 data points and the sequences include 3-17 POIs. The MDC dataset contains many longer sequences compared to the StudentLife dataset. However, the range of the POI number (3-18 in most cases) in the sequences from the MDC dataset is similar with that from the StudentLife dataset, which is reasonable.

\subsection{Incremental-Prediction Accuracy and Times of Winning}

To simulate the real-time performance of the selected methods, we used Incremental-Prediction Accuracy (IPA) as an evaluation metric. From the beginning of each sequence, we predict the next place based on the previous information. The models in each method will be updated once a new data point is available. The $I P A$ is calculated as in Equation 4, where $n_{\text {correct }}(t)$ is the number of correct predictions up to the current moment $t$ and $n_{\text {total }}(\boldsymbol{t})$ is the number of total predictions up to $t$.

$$
P(t)=\frac{n_{\text {correct }}(t)}{n_{\text {total }}(t)}
$$

In some related work, conventional validation (i.e. partitioning the data set into two sets, e.g. $70 \%$ for training and $30 \%$ for testing) is used $[2,14]$. However, this method does not allow analyzing the performance over time from the beginning to the end of data collection. Cross-validation is also not suitable for sequential prediction, especially when using sequential patterns (e.g., $\mathrm{MC1}$ and Active LeZi), because the sequential information of human behavior will be broken when the sequence is segmented or sampled.

Along with IPA, we introduce another metric called Times of Winning (ToW) as shown in Formula (5). $P_{m}(t)$ means the IPA of a given method, while $\left\{P_{\varnothing}(t)\right\}$ indicates the IPA of each used method. Therefore, ToW refers to the frequency of the cases where a method beats others over the given sequence.

$$
\text { ToW }_{m}=\text { number of }\left(P_{m}(t)>\left\{P_{\varnothing}(t)\right\}\right)
$$

\subsection{Predictability Limit}

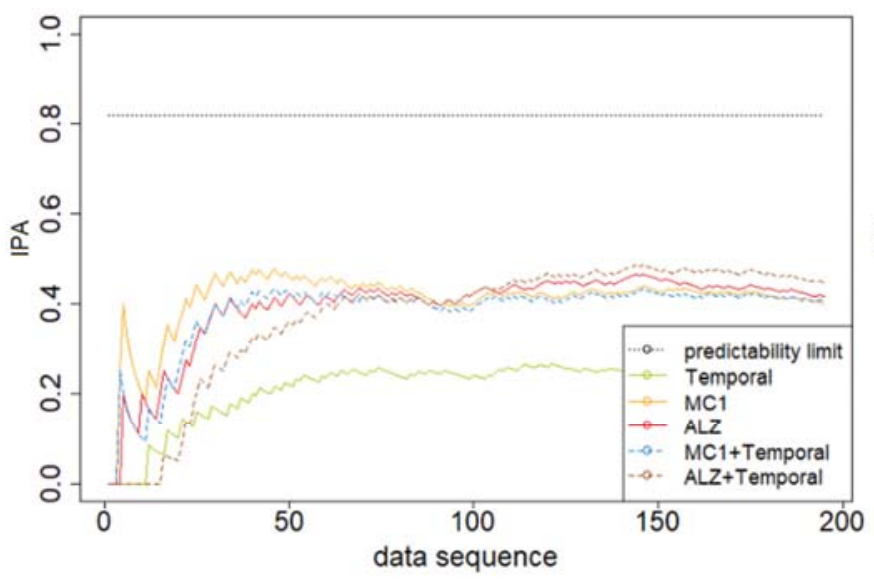

To compare the selected methods, we are not only interested in the relative performance of the methods. Instead, we also examine their distance to the predictability limit. The upper limit of human mobility predictability represents the average probability of correctly predict the next POI that can be best achieved, given a POI sequence. Depending on the entropy rate of a given sequence, the predictability limit can be estimated. Song et al. [23] provided an approach for estimating the theoretical predictability limit of sequential data using LZ-based entropy rate estimation [12] and Fano's inequality [7]. The predictability limit provides us with a theoretical benchmark for estimating how good a prediction method works.

\subsection{Accuracy@K}

To analyze the average accuracy of each single pattern-based method, we also use the Accuracy@K metric which indicates the rate that the top $\mathrm{K}$ prediction candidates contain the correct prediction. This metric has been well used in location prediction and location-based recomentation [29, 31]. In our evaluation, we use $\mathrm{K}$ as 3 .

\subsection{Results}

Before showing the results of the average prediction for all users in each dataset, we first show the IPA of each method for two users' data from each dataset in Figure 4. The figure shows us that the IPAs of all methods fluctuate during a short period of time in the beginning. After this fluctuation, most methods arrive at a relatively stable IPA level with a slight increase as more information is obtained. We can also observe that there are some common trends of short and rapid decreases in the middle of the process, which indicates a change of the users' daily routines. Although the predictability limit (the horizontal black dotted lines in Figure 4) is only subject to the sequential pattern-based prediction, it also cannot be reached by other methods. For both users, the temporal pattern-based method (green line in Figure 4) performs the worst, while the best-performing method changes over time.

The average prediction accuracies of each method for both datasets are shown in Figure 5. All the reported numbers

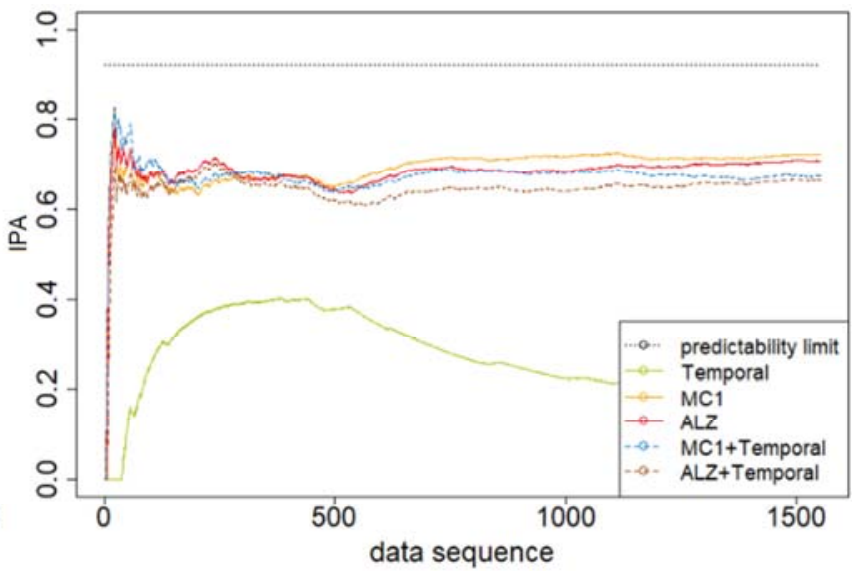

Figure 4: The results of incremental-prediction accuracy (IPA) for user \#2 from StudentLife dataset (left) and user \#8 from MDC dataset (right). 
represent the median values in the remainder of this section. For the StudentLife dataset, the results of method (2) (MC1) and method (3) (ALZ) are comparable (0.41), while being significantly better than method (1) $(0.22)$, (4) (0.30), and (5) (0.26). When comparing only the two sequential pattern-based methods, method (2) (MC1) performs slightly better than method (3) (ALZ) in the perspectives of the interquartile range and whiskers of the box plots. Combining temporal and sequential patterns cannot improve the average prediction accuracy, which is out of our expectation. For the MDC dataset, method (3) (0.71) outperforms method (2) (0.62), which is different than the result for the StudentLife dataset. The temporal pattern-based methods still performed the worst, and the average prediction accuracy of the methods combining temporal and sequential patterns was worse than the methods using single sequential pattern. All selected methods were far from reaching the predictability limits (PL) for both datasets.
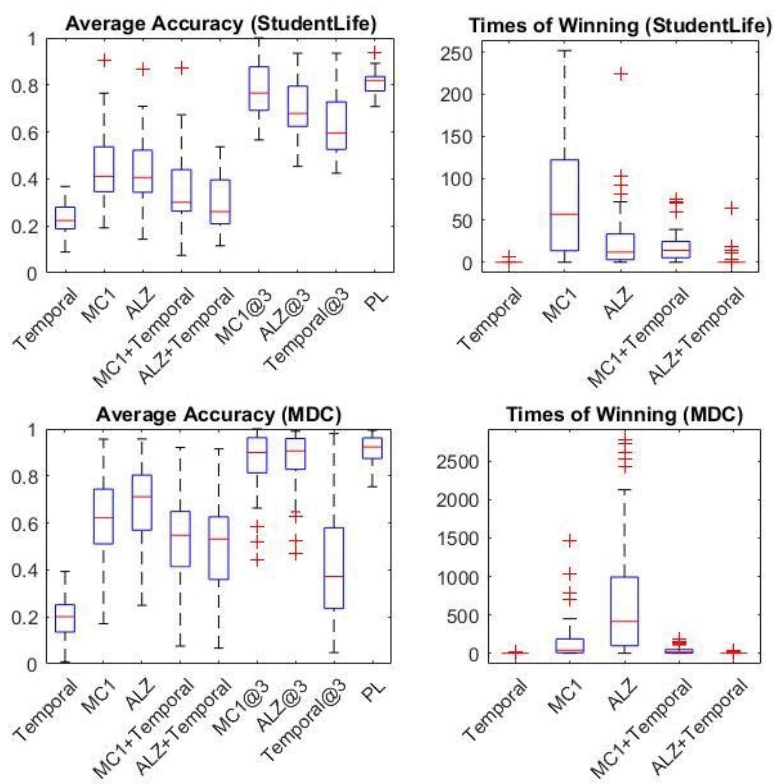

Figure 5: The average accuracy and Times of Winning (ToW) of each method for the StudentLife and MDC datasets. ALZ presents Active LeZi and PL denotes predictability limit.

Given our results so far, research question Q1 can be answered by stating that the selected sequential pattern-based methods outperform the selected temporal pattern-based method. From the view of average accuracy, the hybrid methods do not perform better than their counterparts that only used sequential patterns. In Figure 4, we already saw that the winner (i.e., the method with the highest IPA in real-time) changes over time.

From the perspective of ToW, method (4) (MC1+Temporal) is best for the StudentLife dataset, while method (3) (ALZ) outperforms the others for the MDC dataset. Therefore, to answer research question Q2, we argue that combining sequential with temporal patterns does not necessarily improve the average prediction performance but can outperform other methods only for some time periods for specific users.
In addition, research question Q3 can be answered by our observation that there is no consistently winning method among the evaluated methods. When considering ToW, method (1) (Temporal) and method (5) (Temporal+ALZ) cannot beat the other methods for most time intervals for both datasets.

In terms of the Accuracy@3 results, we can see that MC1@3 achieves the highest accuracy $(0.77)$ in the StudentLife dataset, while ALZ@3 (0.93) performs slightly better than MC1@3 (0.92) for the MDC dataset, as shown in Figure 5. If we compare each @3 3 accuracy of $\mathrm{MC} 1$ and Active LeZi with their top-1 prediction accuracy respectively, MC1@3 improves more than ALZ@3 for both datasets.

\section{THE VISUALIZATION TOOL UP $\mathrm{UP}^{3} \mathrm{~V}$}

Only using the IPAs of the tested methods at the average-level of individuals is not enough to help developers and system designers answer the question: Which method(s) shall we choose in realworld personalized applications, e.g. JITAIs? This question is precisely the aim of research questions Q4. To help experts analyze the performance of each method at the level of the individual, as well as at the POI level, we developed a visualization tool, which we termed the User's Pattern and POI Prediction Visualizer $\left(U P^{3} V\right)$. It interactively visualizes the results for each user's data. The target users of this tool are the experts who analyze human mobility patterns. This proof-ofconcept prototype is implemented based on Shiny ${ }^{1}$ in an RStudio environment. Leaflet ${ }^{2}$ and graphics ${ }^{3}$ were used for the map and charts.

Figure 6 shows $U^{3} \mathrm{~V}$ containing the map with spatial information (left side) and the information panel (right side), which contains the statistical visualization showing temporal, as well as sequential results. Once a POI - represented as a cluster of dots in a specific color on the map - is selected, all the transitions from other places to the selected POI will be shown on the map. The information panel contains charts presenting prediction results at the level of each user (as shown in Figure 4) and each POI (see chart (1) in Figure 6). To support temporal patterns analysis, two charts are generated (chart (2) and (3)) presenting the arrival time distribution per weekday of the selected POI and the stay periods for the selected POI in the visualization tool. Chart (2) and (3) can support experts to discover temporal patterns. For example, from chart (3) we can deduce that the selected POI should be the home of the user, because the user typically remained in this place during the night (the yellow cluster on the map). Further, from chart (2), we can see that the arrival time at this place was widely distributed for different days of the week, e.g. the most frequent arrival time on Wednesdays was close to midnight.

\footnotetext{
https://shiny.rstudio.com/

https://rstudio.github.io/leaflet/

https://www.r-graph-gallery.com/
} 


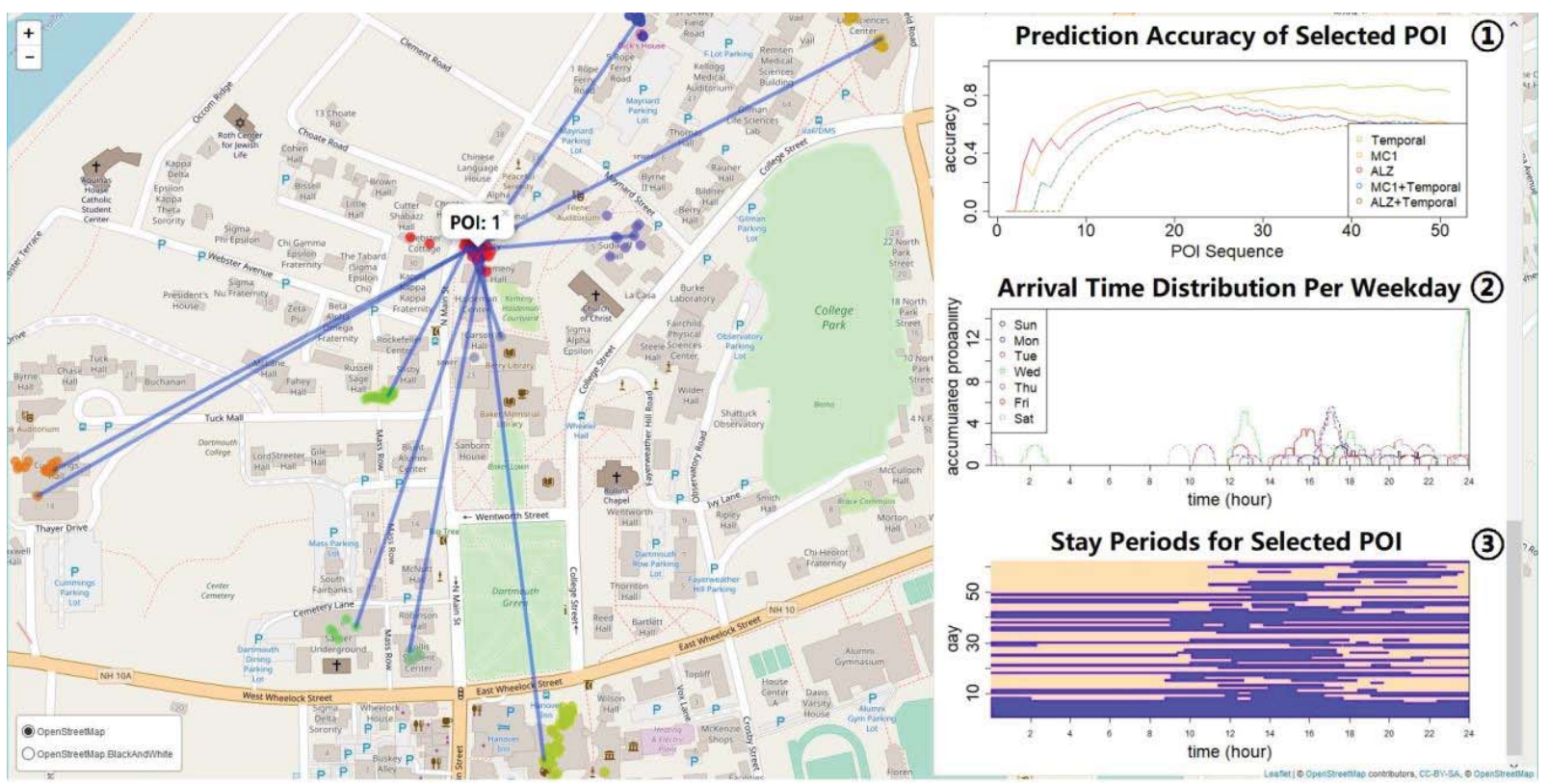

Figure 6: User's Pattern and POI Prediction Visualizer (UP $\left.{ }^{3} V\right)$. The map (left side) displays the places/spatial information and an information window (right side) shows the results per selected user. The information panel shows for the selected POI:

(1) the prediction accuracy, (2) the arrival time distribution per weekday, and (3) the stay periods.

The panel also has a data selector for switching the dataset and the user ID. When the dataset or user ID is selected, the map and the figures update accordingly. When any POI is clicked, the corresponding marker will appear on the map and the result of incremental-prediction accuracy in the POI level will show up as in chart (1) in Figure 6, which helps us answer research question Q4. Using $U P^{3} \mathrm{~V}$, we found that the accuracies of different method vary depending on the current POI of a specific user. Furthermore, the result shows that the performance of different methods changes and the winner varies over time for a selected POI.

Using this visualization tool, an expert can gain a deeper understanding of each user's data in terms of the information available on spatial, temporal, and sequential patterns for each POI. We find that no method can consistently outperform other methods for all places visited by a certain user. For sequences of most users, the winner changes over time. Besides, given a time point over the sequence of a user, we find that the winning method for a specific POI (see chart (1) in Figure 6) can be different from the winning method for all POIs on average for the user (see Figure 4).

\section{DISCUSSION}

Based on the initially mentioned research questions, the basic idea of this work was to test if integrating temporal and sequential patterns using joint conditional probability is able to improve the prediction accuracy. Our results did not show performance improvement on average. However, the hybrid methods (4) and
(5) can beat single sequential pattern-based methods (2) and (3) for some periods of the data sequence in terms of incrementalprediction accuracy. When considering the temporal pattern-based method we adopted, the performance was quite low. Using our visualization tool, we plan to test more methods based on temporal patterns in the near future.

Another interesting finding was that the more sophisticated sequential patterns (e.g., Active LeZi) were not always more effective than simple ones (e.g., MC1). In addition, the performance of the algorithms highly depends on the corresponding dataset. Based on two datasets (128 users in total), our methods focused on three single patterns (two sequential patterns and one temporal pattern). Larger datasets may change the results of prediction at the average level, but the findings at the level of the individual and at the POI level using IPA and ToW will still stand, which will be relevant for future approaches. In the following, the implications based on the results of our evaluation and the discoveries made with $\mathrm{UP}^{3} \mathrm{~V}$ are summarized:

(1) We suggest that methods based on different patterns are used in parallel for next-place prediction because individuals' mobility data is quite diverse over time. JITAI designers can always choose the winning method based on incrementalprediction accuracy to take full advantage of different methods. If several POIs are examined, using the winning method over time for the corresponding POIs is recommended instead of using the winning method for all POIs on average.

(2) When using top 3 predictions (Accuracy@K), the prediction accuracy can be much higher than for top 1 prediction when using $\mathrm{MCl}$ or Active LeZi. 
(3) From our observation using $U P^{3} V$, we found that the stay periods of some POIs also have embedded patterns, which can be potentially used for improving the prediction accuracy.

\section{CONCLUSION}

In this paper, we addressed the problem of next-place prediction to provide insights on related mobile health applications. Based on individuals' sequential and temporal patterns in the mobility data collected by smartphones, we posed four research questions regarding the effectiveness of three single pattern-based methods (first-order Markov chain, Active LeZi, and an arrival time distribution-based temporal pattern) and two methods using joint conditional probability to combine temporal and sequential patterns. Our primary contributions include: (1) we evaluated the performance of combining sequential and temporal patterns when compared to the use of single patterns, (2) we used the predictability limit to compare the prediction performance of the selected methods, (3) we investigated the performance of nextplace prediction methods at the level of the individual and at the level of POIs (Places of Interest) using incremental prediction accuracy and times of winning.

We evaluate the five selected methods at both of these levels for two publicly available datasets. Using incremental prediction accuracy, we find that each of the selected methods performs differently among users and POIs over time. On average, the sequential pattern-based methods perform better than the temporal pattern-based methods. Using joint conditional probability to integrate different patterns is effective to some extent but does not outperform single pattern-based methods on average. In addition, the top 3 predictions (Accuracy@K) of sequential pattern basedmethods show high accuracy. Our results suggest that using multiple methods in practice, and that relying on the best performing method for each individual and even for each POI depending on incremental prediction accuracy, can lead to an optimal outcome.

In addition, the visualization tool $\mathrm{UP}^{3} \mathrm{~V}$ was presented, which is designed to analyze the prediction results per user and per POI. In future work, we plan to extend and evaluate our visualization tool with domain experts. Additionally, we will compare more temporal and sequential patterns and explore how to effectively combine these patterns.

\section{REFERENCES}

[1] Bhattacharya, A. and Das, S.K. 2002. LeZi-Update: An Information-Theoretic Framework for Personal Mobility Tracking in PCS Networks. Wireless Networks. 8, (2002), 121-135.

[2] Burbey, I. and Martin, T.L. 2012. When Will You Be at the Office? Predicting Future Locations and Times. Springer, Berlin, Heidelberg. 156-175.

[3] Cheng, Y. et al. 2017. An improved Markov method for prediction of user mobility. International Workshop on Green ICT and Smart Networking, GISN 2016. (2017), 394-399. DOI:https://doi.org/10.1109/CNSM.2016.7818454.

[4] Cleary, J.G. et al. 1984. Unbounded length contexts for PPM. Proceedings DCC '95 Data Compression Conference. (1984), 52-61. DOI:https://doi.org/10.1109/DCC.1995.515495.

[5] Do, T.M.T. et al. 2015. A probabilistic kernel method for human mobility prediction with smartphones. Pervasive and Mobile Computing. 20, (2015), 13-28. DOI:https://doi.org/10.1016/j.pmcj.2014.09.001.
[6] Do, T.M.T. and Gatica-Perez, D. 2014. Where and what: Using smartphones to predict next locations and applications in daily life. Pervasive and Mobile Computing. 12, (2014), Computing. 12,
DOI:https://doi.org/10.1016/j.pmcj.2013.03.006.

[7] Fano, R.M. 1949. THE TRANSMISSION OF INFORMATION. (1949).

[8] Gopalratnam, K. and Cook, D.J. 2007. Online Sequential Prediction via Incremental Parsing: The Active LeZi Algorithm. IEEE Intellignet Systems. (2007).

[9] Ikanovic, E.L. and Mollgaard, A. 2017. An alternative approach to the limits of predictability in human mobility. EPJ Data Science. 6, 1 (2017). DOI:https://doi.org/10.1140/epjds/s13688-017-0107-7.

[10] Khoroshevsky, F. and Lerner, B. 2016. Human Mobility-Pattern Discovery and Next-Place Prediction from GPS Data. MPRSS. (2016). DOI:https://doi.org/10.1007/978-3-319-59259-6.

[11] Kiukkonen, N. et al. 2010. Towards rich mobile phone datasets: Lausanne data collection campaign. Proceedings ACM International Conference on Pervasive Services (ICPS). Berlin, (2010).

[12] Kontoyiannis, I. et al. 1998. Nonparametric entropy estimation for stationary processes and random fields, with applications to English text. IEEE Transactions on Information Theory. 44, 3 (1998), 1319-1327.

[13] Laurila, J.K. et al. 2012. The mobile data challenge: Big data for mobile computing research. Proceedings of the Workshop on the Nokia Mobile Data Challenge, in Conjunction with the 10th International Conference on Pervasiver Computing. (2012), 1-8. Pervasive Computing. DOI:https://doi.org/10.1016/j.pmcj.2013.07.014.

[14] Lee, S. et al. 2016. Next Place Prediction Based on Spatiotemporal Pattern Mining of Mobile Device Logs. (2016), 1-19. DOI:https://doi.org/10.3390/s16020145.

[15] Liu, Q. et al. 2016. Predicting the Next Location: A Recurrent Model with Spatial and Temporal Contexts. Proceedings of the 30th Conference on Artificial Intelligence (AAAI 2016). (2016), 194-200. DOI:https://doi.org/10.1016/j.egypro.2016.11.209.

[16] Lu, X. et al. 2013. Approaching the Limit of Predictability in Human Mobility. Scientific Reports. 3, 1 (2013), 2923. DOI:https://doi.org/10.1038/srep02923.

[17] Naughton, F. et al. 2016. A Context-Sensing Mobile Phone App (Q Sense) for Smoking Cessation: A Mixed-Methods Study. JMIR mHealth and uHealth. 4, 3 (Sep. 2016), e106. DOI:https://doi.org/10.2196/mhealth.5787.

[18] Petzold, J. et al. 2006. Comparison of Different Methods for Next Location Prediction. (2006), 909-918. DOI:https://doi.org/10.1007/11823285 96.

[19] Poppinga, B. 2014. Sensor-based Identification of Opportune Moments to Trigger Unobtrusive Notifications. IEEE Pervasive Computing. (2014).

[20] Rabbi, M. et al. 2015. MyBehavior: automatic personalized health feedback from user behaviors and preferences using smartphones. Ubicomp '15, September 7-11, 2015, Osaka, Japan. (2015), 707-718. DOI:https://doi.org/10.1145/2750858.2805840.

[21] Rodriguez-Carrion, A. et al. 2012. Study of LZ-based location prediction and its application to transportation recommender systems. Sensors (Switzerland). 12, 6 (2012), 7496-7517. DOI:https://doi.org/10.3390/s120607496.

[22] Scellato, S. et al. 2011. NextPlace: A Spatio-temporal Prediction Framework for Pervasive Systems. Springer, Berlin, Heidelberg. 152-169.

[23] Song, C. et al. 2010. Limits of Predictability in Human Mobility. Science. 327, (2010), 387-392. DOI:https://doi.org/10.1126/science.1177170.

[24] Song, C. et al. 2010. Limits of Predictability in Human Mobility (SM). $\begin{array}{llll}\text { Science. } & 327, & 5968 & \text { (2010), }\end{array}$ DOI:https://doi.org/10.1126/science. 1177170

[25] Wang, R. et al. 2014. StudentLife: Assessing Mental Health, Academic Performance and Behavioral Trends of College Students using Smartphones. Proc. of ACM UbiComp 2014 (New York, New York, USA, 2014), 3-14.

[26] Wang, Y. et al. 2018. Clustering with Temporal Constraints on SpatioTemporal Data of Human Mobility. Unpublished (2018).

[27] Wang, Y. et al. 2016. "Fingerprints": Detecting meaningful moments for mobile health intervention. Proceedings of the 18th International Conference on Human-Computer Interaction with Mobile Devices and Services Adjunct, MobileHCI 2016 (2016).

[28] World Health Organization 2011. mHealth: New horizons for health through mobile technologies. Observatory. 3, (2011), 112. DOI:https://doi.org/ISBN 9789241564250.

[29] Xie, M. et al. Learning Graph-based POI Embedding for Location-based Recommendation. DOI:https://doi.org/10.1145/2983323.2983711.

[30] Ye, Y. et al. 2009. Mining Individual Life Pattern Based on Location History. Mobile Data Management: Systems, Services and Middleware, 2009. MDM'09 (2009), 1-10

[31] Zhang, Z. et al. 2017. NEXT: A Neural Network Framework for Next POI Recommendation. (2017)

[32] Ziv, J. and Lempel, A. 1978. Compression of individual sequences via variable-rate coding. IEEE Transactions on Information Theory. 24, 5 (Sep. 1978), 530-536. DOI:https://doi.org/10.1109/TIT.1978.1055934. 